\title{
0361 DEVELOPING GLOBAL AND REGIONAL ESTIMATES OF FATAL INJURY RELATED TO WORK
}

T Driscoll*, C Bryan-Hancock Correspondence: Sydney School of Public Health, Building A27 University of Sydney, Australia

10.1136/ip.2010.029215.361

Aims To develop revised global and regional estimates of the burden of fatal injury resulting from work-related exposures as part of the new Global Burden of Disease (GBD) initiative. This work involves calculating and comparing burden estimates for 2005 and 1990.

Methods The approach builds on previous GBD injury work and makes use of more recently published data and information from as many appropriate sources as possible. Published data on fatal injury rates in representative countries and regions were identified and reviewed for methodological rigour and relevance. Rates were extrapolated from the most relevant nearby region if necessary. Industry-specific estimates were identified where possible, to take into account differences in risk arising from different industry distributions between regions and over time. Employment information was obtained from standard published sources.

Results Representative rates were found for at least one country in nearly all regions. The main difficulties encountered were deciding which study(s) best represented the regions and times of interest, and identifying and adjusting for different inclusion criteria.

Conclusions Calculating useful global and regional estimates of work-related injury burden raises many challenges but can be achieved by careful assessment of study methodology and extensive searching of potentially relevant data sources. 